\title{
Danish Registry of Childhood and Adolescent Diabetes
}

This article was published in the following Dove Press journal:

Clinical Epidemiology

25 October 2016

Number of times this article has been viewed

\author{
Jannet Svensson' \\ Charlotte Cerqueira ${ }^{2}$ \\ Per Kjærsgaard ${ }^{3}$ \\ Lene Lyngsøe ${ }^{4}$ \\ Niels Thomas Hertel ${ }^{5}$ \\ Mette Madsen ${ }^{6}$ \\ Henrik B Mortensen' \\ Jesper Johannesen' \\ 'Pediatric and Adolescent \\ Department, Copenhagen University \\ Hospital, Herlev and Gentofte, Herlev, \\ ${ }^{2}$ Registry Support Centre (East) - \\ Epidemiology and Biostatistics, \\ Research Centre for Prevention and \\ Health, Capital Region of Denmark, \\ Glostrup, ${ }^{3}$ Pediatric Department, \\ County Hospital Herning, \\ Herning, ${ }^{4}$ Pediatric and Adolescent \\ Department, Nordsjællands Hospital, \\ Hillerød, ${ }^{5} \mathrm{HC}$ Andersen Childrens \\ Hospital, Odense University Hospital, \\ Odense, ${ }^{6}$ Pediatric Department, \\ Aalborg University Hospital, Aalborg, \\ Denmark
}

Correspondence: Jannet Svensson Pediatric and Adolescent Department, Copenhagen University Hospital, Herlev, Herlev Ringvej 75, 2730 Herlev, Denmark Tel +453868 1089

$\mathrm{Fax}+453868510 \mathrm{I}$

Email jannet.svensson@regionh.dk
Aim: The aims of the Danish Registry of Childhood and Adolescent Diabetes (DanDiabKids) are to monitor and improve the quality of care for children and adolescents with diabetes in Denmark and to follow the incidence and prevalence of diabetes.

Study population: The study population consists of all children diagnosed with diabetes before the age of 15 years since 1996. Since 2015, every child followed up at a pediatric center $(<18$ years of age) will be included.

Main variables: The variables in the registry are the quality indicators, demographic variables, associated conditions, diabetes classification, family history of diabetes, growth parameters, self-care, and treatment variables. The quality indicators are selected based on international consensus of measures of good clinical practice. The indicators are metabolic control as assessed by $\mathrm{HbA1}$, blood pressure, albuminuria, retinopathy, neuropathy, number of severe hypoglycemic events, and hospitalization with ketoacidosis.

Descriptive data: The number of children diagnosed with diabetes is increasing with $\sim 3 \%$ per year mainly for type 1 diabetes (ie, 296 new patients $<15$ years of age were diagnosed in 2014). The disease management has changed dramatically with more children treated intensively with multiple daily injections, insulin pumps, and increased number of self-monitored blood glucose values per day. These initiatives have resulted in a significant improvement in HbAlc over the years and a decrease in the number of children experiencing severe hypoglycemia, diabetic nephropathy, and retinopathy.

Conclusion: The systematic collection of data in DanDiabKids documents improved quality of care over the last 12 years, despite a substantial increase in the number of patients cared for by pediatric departments in Denmark, fulfilling the purpose of the registry.

Keywords: DanDiabkids, treatment modalities, HbA1c, severe hypoglycemia, quality of care

\section{Aim of the registry}

The aims of the registry are to improve and monitor the quality of care for children and adolescents with diabetes in Denmark and to follow the incidence and prevalence of diabetes over time. Furthermore, the registry supports and encourages research in the area of childhood diabetes.

\section{Study population}

The study population consists of all children diagnosed with diabetes before the age of 15 years since 1996, including all children followed up by a pediatric department since $1996 .{ }^{1}$ Since 2015 , every child aged up to 18 years admitted to a pediatric center will be included, thereby extending the study population in the future. In 2014, 
328 new patients were included, of whom 296 were $<15$ years of age. Over the years 1996-2014, a total of 5,602 children have been included (Figure 1). In the beginning, only children with type 1 diabetes (T1D) based on a clinical diagnosis were included, and after 2006, children with all types of diabetes were included. There is a continuous merging with the Danish National Patient Register with all International Statistical Classification of Diseases and Related Health Problems-tenth revision (ICD10) codes for diabetes (ie, DE10.x, DE11.x, DE12.x, DE13.x, DE14.x). Once a year, all patients in the National Patient Register not found in the Danish Registry of Childhood and Adolescent Diabetes (DanDiabKids) are validated to ensure that all children with diabetes are registered in DanDiabKids.

\section{Main variables}

The demographic variables include the municipality at onset, ethnicity, sex, and age at onset of diabetes. The completeness

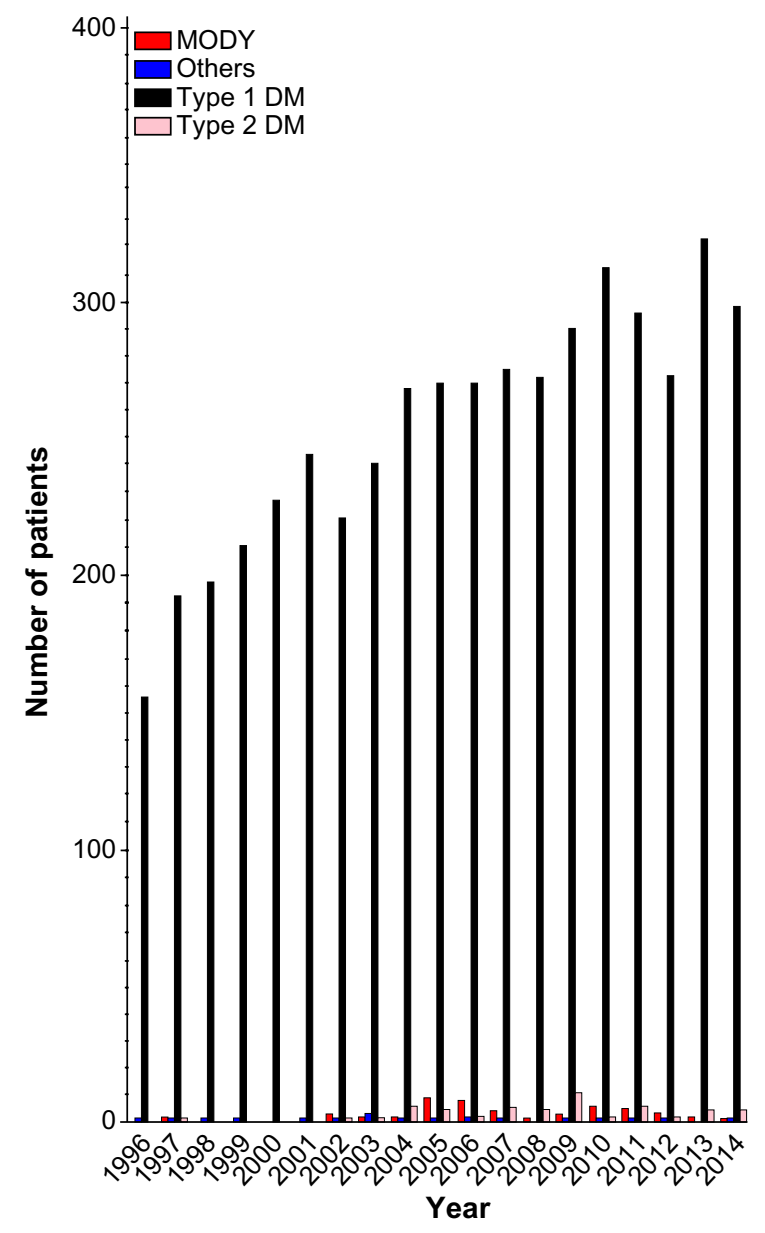

Figure I Newly diagnosed patients per year.

Notes: The number of patients diagnosed per year categorized according to the latest classification. MODY is based on genetic tests. Some individuals were initially diagnosed as type I diabetes but later reclassified due to new genetic tests. The ascertainment is $>99 \%$ per year.

Abbreviations: MODY, maturity onset diabetes of the young; DM, diabetes mellitus. of data is $100 \%$, except for ethnicity, where $<1 \%$ were missing in 2014.

The classification is primarily based on a clinical presentation, but it is possible to register genetics, stimulated C-peptide, and autoantibody titers if measured. Family history of diabetes is recorded only at onset.

The main variables for monitoring and improving quality of care in the registry are the quality indicators. ${ }^{2,3}$ The indicators are selected based on international consensus of measures of good clinical practice. All indicators are defined based on international standards. ${ }^{2,4}$ The outcome indicators are metabolic control expressed as glycated hemoglobin (HbA1c), blood pressure (BP), albuminuria, retinopathy, neuropathy, number of severe hypoglycemic events, and hospitalization with ketoacidosis (Figure 2). Once a year, $>90 \%$ of the participating centers send one HbAlc sample to Copenhagen University Hospital at Herlev. The Department of Pediatrics at Copenhagen University Hospital has its own specialized laboratory where the determination of $\mathrm{HbA} 1 \mathrm{c}$ is performed for all participating centers in Denmark according to the International Federation of Clinical Chemistry, whereas onethird of locally measured $\mathrm{HbA} 1 \mathrm{c}$ are determined with a nonstandardized immunoturbidimetric assay (the DCA Vantage ${ }^{\circledR}$ Analyzer [DCA 2000 HbA1c system, Bayer AG, Leverkusen, Germany]). BP was first selected as an outcome indicator in 2013; this variable has not yet been validated. Guidelines for measuring BP follow international standards. The data completeness for BP was $87 \%$ in 2014 and is anticipated to increase in the coming years, where a national clinical

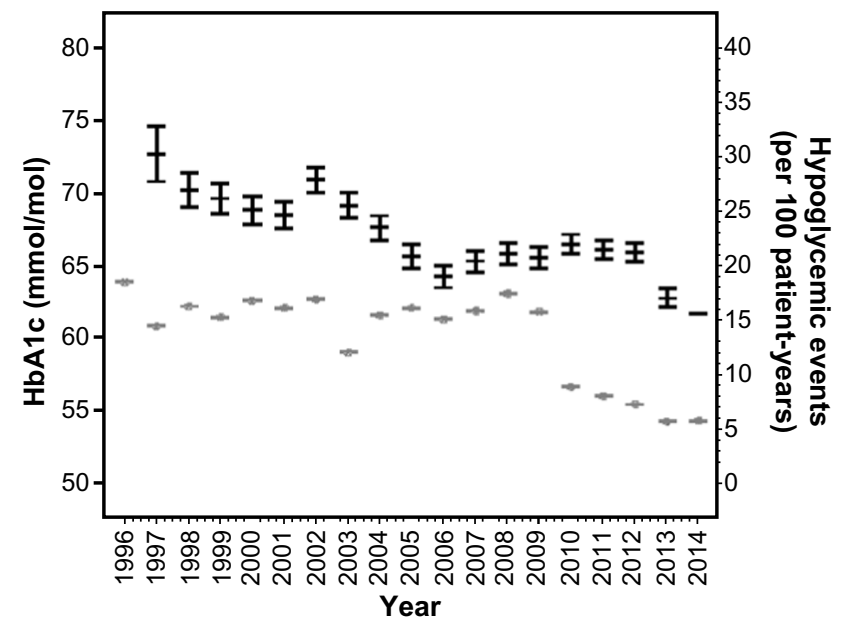

Figure $2 \mathrm{HbAlc}$ and severe hypoglycemic events (per 100 person-years). Notes: The fluctuation in $\mathrm{HbAlc}$ (bars) and the incidence of severe hypoglycemic events (unconsciousness or seizures [circles]) during the last 12 years in children diagnosed with type I diabetes before the age of 15 years. $\mathrm{HbAlc}$ is in $\mathrm{mmol} / \mathrm{mol}$ (bars), whereas the incidence of hypoglycemia (IR) is number per 100 person-years (circles). Data completeness increased from 1996 to 2000 and then stabilized above $75 \%$; since 2006, data completeness has exceeded $80 \%$.

Abbreviations: IR, incidence rates per 100 person-years; HbAIc, glycated hemoglobin. 
guideline of at least $95 \%$ being screened will be implemented. Presently, a definition of hypertension is not included because we have not had access to calculation of a percentile/standard deviation (SD) score. This is now available, and an indicator defining the acceptable number of children with hypertension, who have not started pharmacological treatment, will be defined. For most variables, data completeness is $>75 \%$ since 2000 and $<20 \%$ is missing from 2006 onward.

The number of severe hypoglycemic events (loss of consciousness/seizures) is recorded at each visit in the outpatient clinic and summarized to the annual status for each individual. The episodes have to be confirmed with a measured blood glucose $<3.5 \mathrm{mmol} / \mathrm{L}$ or consciousness being reestablished after glucagon or IV glucose infusion, to exclude epilepsy or other medical reasons for seizure. If the number of hypoglycemic events exceeds ten per year, the pediatric center is contacted to confirm whether the number is correct. The number of hospitalizations with ketoacidosis is recorded similarly and summarized once yearly. The bicarbonate or $\mathrm{pH}$ at admission has to be registered in order to include only those with confirmed ketoacidosis - defined as standard bicarbonate $<15 \mathrm{mmol} / \mathrm{L}$ or $\mathrm{pH}<7.3$. There were $\sim 5 \%$ missing registrations of hypoglycemia and 7\% missing registrations of ketoacidosis in 2014. These numbers have been stable in the last 3-5 years.

Screening and monitoring for microvascular complications (retinopathy, nephropathy, neuropathy) are initiated at 12 years, 15 years, and 18 years of age. Before 2010, screening for microalbuminuria was based on timed overnight urinary albumin excretion rate $(>20 \mu \mathrm{g} / \mathrm{min})$. Due to insufficient data completeness, the screening procedure was changed to a spot urine (albumin/creatinine ratio). If negative, this is sufficient, but if positive $(>2.5 \mathrm{mg} / \mathrm{mmol}$ in males and $>3.5 \mathrm{mg} / \mathrm{mmol}$ in females), two additional overnight measures of albumin excretion are required. At least two positive measures of albumin excretion within a period of 6 months are necessary to confirm microalbuminuria. The change in the procedure has led to increased data completeness. There was a screening completeness of $93 \%$ in 2014. Screening for retinopathy is accepted either as eye fundus examination (fundoscopy) or by two-field $40^{\circ}-60^{\circ}$ fundus photography. Most of the retinopathies are screened by fundus photography. Screening completeness shows huge variation across regions, with $80 \%$ at the national level, indicating potentials for improvement. Missing screening is sometimes due to the fact that some of the patients are screened by an ophthalmologist or in an eye-care center outside the hospital, and information regarding the examinations is not returned to the primary clinic. Neuropathy is screened using biothesiometry. There is no clear international definition of neuropathy in children, and the challenge is that the threshold values for vibration sense increase with linear height. So far, we have used adult limits for neuropathy of $20 \mathrm{~V}$, which is too coarse and leads to none of the children being diagnosed with neuropathy. The plan is to define limits for children below and above $150 \mathrm{~cm}$ of height based on measures in healthy children.

Growth is measured annually by linear height, body weight, and body mass index. Outliers, defined as values of 3SD from the mean, are validated by sending patient lists to the departments for confirmation. Furthermore, it is impossible to register a height lower than previous measures in the registry.

A
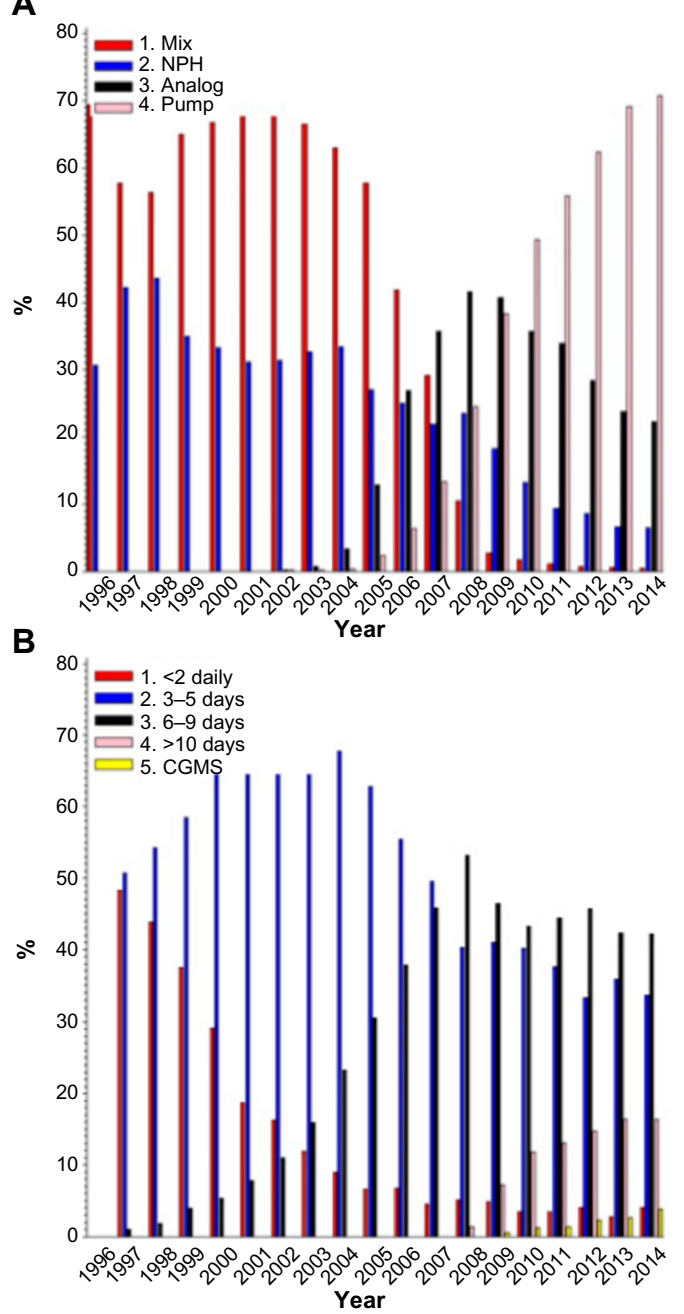

Figure 3 Treatment modalities and glucose monitoring.

Notes: (A) The number of children treated with different insulin types or insulin pumps per year. Data completeness increased from 1996 to 2000 and thereafter stabilized above $75 \%$; since 2006 , it has been stable above $80 \%$. (B) The number of SMBG per day registered for children with diabetes per year or the number using CGMS > I week per month. Using CGMS > I week per month overrules the number of SMBG. Data completeness increased from 1996 to 2000 and thereafter stabilized above $75 \%$; since 2006 , is has been stable above $80 \%$.

Abbreviations: CGMS, continuous glucose monitoring; SMBG, self-monitored blood glucose values; NPH, neutral protamine hagedorn. 
The treatment and self-care are recorded annually, including injection methods, insulin types, number of injections or boluses, and self-monitoring of glucose (Figure 3). There are $5 \%-10 \%$ missing data on these variables during the recent years.

T1D is associated with other autoimmune manifestations, especially celiac and thyroid disease. It is recommended that celiac disease is screened annually for the first 3 years after diagnosis and thereafter in case of symptoms. Celiac disease and thyroid disease are not mandatory variables in the registry, but all centers are recommended to follow international guidelines regarding screening for thyroid diseases, which means screening at onset and every second year thereafter, if no obvious signs of thyroid disease are present.

The standard of each quality indicator and registration practice of variables is discussed at annual meetings, where results comparing different centers are discussed. Each year, a subset of variables is in focus. Currently, a clear, practical, and clinical-oriented definition and screening procedure for microvascular complications and hypertension are in focus.

\section{Follow-up}

The registry was initiated in 1996, and since then, there has been an annual screening and monitoring for microvascular complications. The registry is run by the steering committee for the Danish Study Group of Childhood Diabetes. From 2006 to 2011, each center twice a year received a list with lacking variables for the indicators, allowing for each center to be aware of missing variables. Unfortunately, since the work with the annual report has been centralized, the centers have not had access to lists of missing values influencing data completeness. These lists have now been published in January 2016.

\section{Examples of research}

Based on the register data, the incidence of T1D in children is found to increase with $>3 \%$ a year, ${ }^{5}$ whereas the increase of type 2 diabetes in the young people is nonexisting in Denmark. ${ }^{6}$ We have demonstrated that metabolic control has improved $^{7}$ and severe hypoglycemic events have decreased. ${ }^{89}$ Furthermore, diabetic ketoacidosis at disease onset is associated with worse metabolic control and higher daily insulin requirement for equal $\mathrm{HbA1c}$ values (ie, higher insulin doseadjusted [IDA]A1c) ${ }^{10}$ several years after diagnosis. ${ }^{11}$

The treatment with insulin pumps and the influence of pump treatment on glycemic control have also been evaluated. We found a significantly better $\mathrm{HbAlc}$ in pump users compared to children on multiple daily insulin injections but a parallel increase in $\mathrm{HbAlc}$ with longer diabetes duration. ${ }^{12,13}$

In collaboration with the Swedish, Norwegian, and Icelandic registries, we have investigated the influence of ethnicity on metabolic control and found a slightly higher $\mathrm{HbAlc}$ in those of nonnative ethnicity, though the difference differed between countries and was less than that seen previously. ${ }^{14}$

The prevalence of celiac disease in healthy children and children with T1D in Denmark and Sweden has been investigated by the presence of autoantibodies. ${ }^{15}$ More Swedish children with T1D were positive compared to the Danish children. However, the surprising finding was a relatively high prevalence of celiac antibodies in $2.8 \%$ of healthy Danish population compared to $0.3 \%$ of the Swedish population.

\section{Administrative issues and funding}

The registry was developed in 1995 by Professors Knut Borch-Johnsen and Henrik B Mortensen in collaboration with the diabetologists working at pediatric departments treating children with diabetes. The development of the register was initially funded by Danish Pharmaceutical Association and Novo Nordisk A/S. Later, the yearly costs were covered by the Copenhagen County. At present, the steering committee has access to funding of $\sim € 10,000$ annually to cover expenses for validation, comments of annual reports, and data export. Since 2011, DanDiabKids has been collaborating with the Adult Diabetes Registry and Diabetic Eye Registry together in the Danish Diabetes Database. Before 2012, DanDiabKids independently prepared the annual reports, and statistical and epidemiologic expertise were funded by "The Danish Study Group on Childhood Diabetes". ${ }^{1}$ Since 2012, this work has been centralized under the Danish Diabetes Database.

\section{Conclusion}

Despite a higher workload treating the increasing number of children with diabetes in Denmark, the pediatric departments in Denmark have managed to improve patient outcome, that is, lowering of HbAlc and a decrease in episodes with severe hypoglycemia. This is likely to lead to a substantial reduction in morbidity and mortality for young adults with diagnoses of diabetes in childhood. The quality-focused registry has fulfilled the purpose of monitoring and following the prevalence and incidence of the disease and has led to improved quality of care in children and adolescents with diabetes in Denmark. 


\section{Acknowledgments}

We thank all members of the Danish Study Group of Childhood Diabetes for their skillful work with definition of standards and selection of outcome indicators in the registry as well as the daily work with registration of each child with diabetes in Denmark. This paper was funded by the Program for Clinical Research Infrastructure (PROCRIN) established by the Lundbeck Foundation and the Novo Nordisk Foundation and administered by the Danish Regions. This study was conducted on behalf of the Danish Study Group of Childhood Diabetes.

\section{Disclosure}

JS has Novo Nordisk shares and is a member of Bayer and Novo Nordisk advisory board. PK has received salary in a Novo Nordisk-sponsored register study on growth hormone treatment. NTH has received fee/salary for lectures for Novo Nordisk, Medtronic, Sanofi-Aventis, and Pfizer. HBM has Novo Nordisk shares and is a member of Novo Nordisk and Boehringer Ingelheim advisory boards. CC, MM, LL, and $\mathrm{JJ}$ report no conflicts of interest in this work.

\section{References}

1. Mortensen HB. Dansk register for børne- og ungdomsdiabetes med tilhørende biobank [Danish childhood diabetes registry combined with a biological bank]. Ugeskr Laeg. 1996;158:358. Danish.

2. Nordly S, Jørgensen T, Andreasen AH, Hermann N, Mortensen HB. Quality of diabetes management in children and adolescents in Denmark1. Diabet Med. 2003;20:568-574.

3. Nordly S, Mortensen HB, Andreasen AH, Hermann N, Jørgensen T. Factors associated with glycaemic outcome of childhood diabetes care in Denmark. Diabet Med. 2005;22:1566-1573.

4. Hanas R, Donaghue KC, Klingensmith G, Swift PGF. ISPAD clinical practice consensus guidelines 2009 compendium. Introduction. Pediatr Diabetes. 2009;10(suppl 12):1-2.
5. Svensson J, Lyngaae-Jørgensen A, Carstensen B, Simonsen LB, Mortensen HB; Danish Childhood Diabetes Registry. Long-term trends in the incidence of type 1 diabetes in Denmark: the seasonal variation changes over time. Pediatr Diabetes. 2009;10:248-254.

6. Oester IMB, Kloppenborg JT, Olsen BS, Johannesen J. Type 2 diabetes mellitus in Danish children and adolescents in 2014. Pediatr Diabetes. 2015.

7. Svensson J, Johannesen J, Mortensen HB, Nordly S; The Danish Childhood Diabetes Registry. Improved metabolic outcome in a Danish diabetic paediatric population aged 0-18 yr: results from a nationwide continuous Registration. Pediatr Diabetes. 2009;10:461-467.

8. Johansen A, Kanijo B, Fredheim S, et al; Danish Society for Diabetes in Childhood. Prevalence and predictors of severe hypoglycemia in Danish children and adolescents with diabetes. Pediatr Diabetes. 2015;16(5):354-360.

9. Fredheim S, Johansen A, Thorsen SU, et al; Danish Society for Diabetes in Childhood and Adolescence. Nationwide reduction in the frequency of severe hypoglycemia by half. Acta Diabetol. 2015;52(3):591-599.

10. Max Andersen ML, Hougaard P, Pörksen S, et al. Partial remission definition: validation based on the insulin dose-adjusted $\mathrm{HbA1c}$ (IDAA1C) in 129 Danish children with new-onset type 1 diabetes. Pediatr Diabetes. 2014;15:469-476.

11. Fredheim S, Johannesen J, Johansen A, et al; Danish Society for Diabetes in Childhood and Adolescence. Diabetic ketoacidosis at the onset of type 1 diabetes is associated with future HbA1c levels. Diabetologia. 2013;56:995-1003.

12. Olsen B, Johannesen J, Fredheim S, Svensson J; The Danish Society for Childhood and Adolescent Diabetes. Insulin pump treatment; increasing prevalence, and predictors for better metabolic outcome in Danish children and adolescents with type 1 diabetes. Pediatr Diabetes. 2015; 16:256-262.

13. Overgaard Ingeholm I, Svensson J, Olsen B, et al; DSBD. Characterization of metabolic responders on CSII treatment amongst children and adolescents in Denmark from 2007 to 2013. Diabetes Res Clin Pract. 2015;109(2):279-286.

14. Fredheim S, Delli A, Rida H, et al. Equal access to health care may diminish the differences in outcome between native and immigrant patients with type 1 diabetes. Pediatr Diabetes. 2014;15:519-527.

15. Adlercreutz EH, Svensson J, Hansen D, et al. Prevalence of celiac disease autoimmunity in children with type 1 diabetes: regional variations across the Øresund strait between Denmark and southernmost Sweden. Pediatr Diabetes. 2015;16(7):504-509.
Clinical Epidemiology

\section{Publish your work in this journal}

Clinical Epidemiology is an international, peer-reviewed, open access, online journal focusing on disease and drug epidemiology, identification of risk factors and screening procedures to develop optimal preventative initiatives and programs. Specific topics include: diagnosis, prognosis, treatment, screening, prevention, risk factor modification,

\section{Dovepress}

systematic reviews, risk \& safety of medical interventions, epidemiology \& biostatistical methods, and evaluation of guidelines, translational medicine, health policies \& economic evaluations. The manuscript management system is completely online and includes a very quick and fair peer-review system, which is all easy to use. 